\title{
Anonymous Signature Schemes
}

\author{
Guomin Yang ${ }^{1}$, Duncan S. Wong ${ }^{1, \star}$, Xiaotie Deng ${ }^{1}$, and Huaxiong Wang ${ }^{2}$ \\ ${ }^{1}$ Department of Computer Science, \\ City University of Hong Kong, \\ Hong Kong, China \\ \{csyanggm, duncan, deng\}@cs.cityu.edu.hk \\ ${ }^{2}$ Department of Computing, \\ Macquarie University, Australia \\ hwang@ics.mq.edu.au
}

\begin{abstract}
Digital signature is one of the most important primitives in public key cryptography. It provides authenticity, integrity and nonrepudiation to many kinds of applications. On signer privacy however, it is generally unclear or suspicious of whether a signature scheme itself can guarantee the anonymity of the signer. In this paper, we give some affirmative answers to it. We formally define the signer anonymity for digital signature and propose some schemes of this type. We show that a signer anonymous signature scheme can be very useful by proposing a new anonymous key exchange protocol which allows a client Alice to establish a session key with a server Bob securely while keeping her identity secret from eavesdroppers. In the protocol, the anonymity of Alice is already maintained when Alice sends her signature to Bob in clear, and no additional encapsulation or mechanism is needed for the signature. We also propose a method of using anonymous signature to solve the collusion problem between organizers and reviewers of an anonymous paper review system.
\end{abstract}

\section{Introduction}

Digital signature is one of the most important primitives in public key cryptography. It is a very useful tool for providing authenticity, integrity and nonrepudiation while it has seldom been considered to provide user privacy by its own. In many applications such as e-voting, e-auction, authentication protocols, and many others, we need to protect a signer's identity from being known by eavesdroppers or other parties in a system. For example, in an anonymous electronic transaction processing system [1] or an anonymous key exchange protocol [18, additional mechanisms or encapsulation techniques such as extra layers of encryption are applied onto their underlying signature schemes for protecting the signer's identity. In some other examples such as [6], several requirements for the signer anonymity of a signature scheme are informally given. However,

\footnotetext{
* The work was supported by a grant from the Research Grants Council of the Hong Kong Special Administrative Region, China (RGC Ref. No. CityU 1161/04E) and a grant from CityU (Project No. 9360087).
} 
among these solutions or discussions, they usually require significant increase of system complexities or lack formal methodologies for analyzing the level of anonymity being provided to signers. Although it is widely believed that a signature scheme by itself may provide a certain degree of anonymity to its signers, there is no formal treatment on this subject. It is still generally unclear on exactly what conditions that a signature itself can provide anonymity of its signer. Comparing with the progress on the decryptor identity exposure issue of public key encryption schemes [2], it has been far lagged behind on the research of the signer anonymity of signature schemes themselves.

Consider the following example (Fig. 1) which is a key transport protocol proposed by Boyd and Park [6] for a mobile client $A$ to transport a session key $\sigma$ to a server $B$. The protocol is also targeted to provide client anonymity by protecting $A$ 's identity $I D_{A}$ from being known by eavesdroppers.

$$
\begin{aligned}
& A \rightarrow B: P K E_{B}\left(I D_{A}, \sigma, \text { count }\right) \\
& A \leftarrow B: \operatorname{Enc}_{\sigma}\left(\text { count }, r_{B}\right) \\
& A \rightarrow B: \operatorname{Sig}_{A}\left(I D_{B}, h\left(\text { count }, \sigma, r_{B}\right)\right)
\end{aligned}
$$

Fig. 1. Boyd-Park Authenticated Key Transport Protocol

In the first message of the protocol, $A$ encrypts $I D_{A}, \sigma$ and a field count under $B$ 's public key encryption function $P K E_{B}$ which is assumed to be publicly known. This protects $A$ 's identity from being known by eavesdroppers. In the third message of the protocol however, $A$ also needs to generate and send a signature to $B$ in clear. Obviously, to hide the identity of $A$, this signature should not provide any meaningful information about $A$ 's identity to eavesdroppers.

To illustrate some subtleties of making a signature signer anonymous, we describe several potential attacking techniques which can be used to compromise a signer's identity.

Redundant Structure Attack. As remarked by the authors in [6], it is important to make sure that the signature does not contain any "redundant" structure, which can be revealed during the signature verification procedure and does not require the signed message to be known, while such a redundant structure may help an eavesdropper identify the mobile client. For example, a recoverable signature scheme 5] allows the message to be recovered and verified from the redundant structure of such a signature once the correct signature verification function is given. Hence if the signature scheme $\operatorname{Sig}_{A}$ in the protocol above is recoverable, an eavesdropper can find out the identity of $A$ by trying the signature verification functions of all mobile clients one by one until a message starting with $I D_{B}$ is recovered and verified.

Different Domain Attack. In order to prevent Redundant Structure Attack, a signature scheme which appears to be immune from such an attack, an ElGamal or Schnorr [16] type signature scheme was chosen for this key transport protocol 6]. However, we notice that an eavesdropper may still be able to 
identify the mobile client by examining the signature from another aspect: simply from the length of a signature. Suppose there are two mobile clients in the system and one of them is communicating with the server using this anonymous key transport protocol. When Schnorr signature scheme is used, the two mobile clients may select their own keys in different groups that could have different sizes. By examining the length of the signature in the protocol, the eavesdropper can tell which mobile client is communicating with the server.

Sparse Message Attack. For signature schemes where redundant structure does not exist and all signers have the same signature domain, an adversary may still be able to find out the signer from just the given signature. Below is an example.

Consider a trapdoor one-way permutation family indexed by signers' public keys (e.g. RSA [15]), a signature of a message is generated by computing the permutation inverse of the message using a signer's private signing key (i.e. a trapdoor information). If the message space is sparse in the image of the permutation family (e.g. the image of the permutation family contains only a few meaningful messages), the adversary is able to find out who the actual signer is. Given a signature, the adversary can find out the actual signer's identity using the following elimination method: For a trial signer, the adversary computes the one-way permutation of the signature indexed by the signer's public key and checks if the result is in the corresponding message space. If it is not, then the adversary is sure that this signer is not the actual signer of the signature. The adversary will simply repeat this elimination procedure until a signer is found.

Contributions. We formally introduce signer anonymous digital signature and define two security models subsequently for it. The first one is static, it provides an intuitive way to screen off signatures which do not have the anonymity property; the second one, a stronger model, combines the static model with the adaptive chosen message attack, and this adaptive model is then used in the security analyses of the signer anonymity of our proposed schemes.

Some commonly used signature schemes are examined. We show that the basic RSA signature scheme [15] is in general not signer anonymous, except in a special case where some restrictive assumptions are applied. We then show that PSS [5] is not signer anonymous even with those restrictive assumptions. We also show that Schnorr and ElGamal signature schemes are not signer anonymous, except all signers are choosing keys under a common domain.

To transform those signature schemes to signer anonymous versions, we propose some extensions of them and show that they are signer anonymous even under our adaptive model. We also propose a new anonymous key exchange protocol which allows a client Alice to establish a session key with a server Bob securely while keeping her identity secret from eavesdroppers. In the protocol Alice sends her signer anonymous signature to Bob in clear, while the anonymity of Alice is already maintained. As another application, we propose a method of 
using anonymous signature to solve the collusion problem between organizers and reviewers of an anonymous paper review system.

Paper Organization. In Sec. 2, we review some related work. This is followed by Sec. 3 in which we introduce a security model for signer anonymous signature. In Sec. 4, we review some commonly used signature schemes and show that they are not signer anonymous. In Sec. 5, we introduce a stronger model for signer anonymous signature and call it the adaptive model. In Sec.6, we propose some modifications of the signature schemes reviewed in Sec. 4 and show their anonymity under the stronger adaptive model. In Sec. 7, we apply our anonymous signature schemes on the design of anonymous key establishment protocols and the construction of an anonymous paper review system which solves the collusion problem between organizers and reviewers.

\section{Related Work}

For the counterpart of digital signature in public key cryptography, the public key encryption with key privacy was introduced and first formalized by Bellare et al. in [2. In their model, a secure key-privacy-enabled encryption scheme not only ensures that an encrypted message is semantically secure against adaptive chosen-ciphertext attacks but also prevents the public from getting the decryptor's identity from the encrypted message. Several techniques were also proposed in 2] for converting a conventional encryption scheme to a key-privacyenabled encryption scheme. However, these techniques cannot be simply applied to digital signature schemes for converting them to anonymous version. The main challenge of constructing an anonymous signature scheme is that signature schemes are not designed for hiding messages. It is different from a public key encryption scheme. For a secure key-privacy-enabled encryption scheme, an attacker (i.e. the one who wants to find out the identity of the decryptor) has access to both the message and the corresponding ciphertext (and of course the public keys of all decryptors in a system). For constructing a secure anonymous signature scheme, on the other hand, we need to consider the impacts of messages to the anonymity of signatures more carefully. For example, if a signature and the corresponding message are given, it is impossible to have a signature scheme be anonymous because the signature is publicly verifiable and the number of public keys in a system is usually limited. Another example, if the message of a challenge signature is not given but the message space is small, it would still be easy to find out the identity of the signer by searching over all the possible messages for each possible signer.

Notice that signer anonymity is not the same as sender anonymity while the latter is not new. In signcryption schemes with key privacy [7, 17, , or in designated verifier signature schemes [12,13, the identity of the sender is protected (i.e. sender anonymity) using the intended decryptor/verifier's public key. Their techniques are similar to that of key-privacy-enabled encryption schemes [2]. An 
anonymous signature scheme, on the other hand, does not have an intended recipient when a signature is generated. It solely focuses on the signer anonymity of a signature scheme itself.

\section{A Static Security Model for Signer Anonymity}

Definition 1. A digital signature scheme is a tuple of four algorithms denoted by $(\mathcal{K}, \mathcal{M}, \mathcal{S}, \mathcal{V})$.

1. The key generation algorithm $\mathcal{K}$ is a randomized algorithm which on input $1^{k}$, where $k \in \mathbb{N}$ is a security parameter, returns in polynomial time a pair (pk,sk) of matching public and secret keys.

2. The message space generator $\mathcal{M}$ is an algorithm which on input a public key $p k$ returns in polynomial time a set $M$ (called the message space with respect to pk). Formally, the output is a description of $M$ and for simplicity, we denote $M$ by $\mathcal{M}(p k)$.

3. The signing algorithm $\mathcal{S}$ is a (possibly randomized) algorithm which on input $1^{k}$, a message $m$ and the secret key sk returns in polynomial time a signature $\sigma$ for $m$.

4. The verification algorithm $\mathcal{V}$ is a deterministic algorithm which on input $1^{k}$, a message $m$, the public key $p k$, and a candidate signature $\sigma$ for $m$ returns in polynomial time a bit indicating the validity of the signature.

(Correctness.) We require that $\mathcal{V}\left(1^{k}, m, p k, \mathcal{S}\left(1^{k}, m, s k\right)\right)=1$ for any $(p k, s k) \leftarrow$ $\mathcal{K}\left(1^{k}\right)$ and $m \in \mathcal{M}(p k)$.

In the following, we specify a basic model which captures our fundamental notion of signer anonymity. For simplicity, we omit the expression of $1^{k}$ from the inputs of $\mathcal{S}$ and $\mathcal{V}$ in the rest of the paper.

\subsection{Static Model}

Definition 2. Let $\mathcal{S D}=(\mathcal{K}, \mathcal{M}, \mathcal{S}, \mathcal{V})$ be a digital signature scheme. Suppose the key generation algorithm is run twice with the security parameterk, and $\left(p k_{0}, s k_{0}\right) \leftarrow$ $\mathcal{K}\left(1^{k}\right)$ and $\left(p k_{1}, s k_{1}\right) \leftarrow \mathcal{K}\left(1^{k}\right)$ are generated. $\mathcal{S D}$ is said to produce computationally indistinguishable signatures (or signatures with signer anonymity in the static model) if for every probabilistic polynomial time (PPT) algorithm $\mathcal{D}$, every positive polynomial $p(\cdot)$, and all sufficiently large $k$ 's,

$$
\begin{array}{r}
\left|\operatorname{Pr}\left[\mathcal{D}\left(1^{k}, p k_{0}, p k_{1}, \sigma_{0}\right)=1\right]-\operatorname{Pr}\left[\mathcal{D}\left(1^{k}, p k_{0}, p k_{1}, \sigma_{1}\right)=1\right]\right| \\
<\frac{1}{p(k)}
\end{array}
$$

where $\sigma_{0} \leftarrow \mathcal{S}\left(m_{0}, s k_{0}\right), \sigma_{1} \leftarrow \mathcal{S}\left(m_{1}, s k_{1}\right)$ and $m_{0} \in_{R} \mathcal{M}\left(p k_{0}\right), m_{1} \in_{R} \mathcal{M}\left(p k_{1}\right)$.

By $x \in_{R} X$, we mean that an element $x$ is randomly chosen from a set $X$. 


\subsection{Discussions}

A message-recoverable signature scheme, such as PSS-R [5], allows the message of each of its signatures to be recovered directly from the signature once the corresponding public key is given while having negligible chance to have a message recovered from the signature if an incorrect public key is supplied. In Def. 2, since public keys are known to $\mathcal{D}$, we can see that a message-recoverable signature scheme cannot be anonymous.

Although messages $m_{0}$ and $m_{1}$ are unknown to the distinguisher $\mathcal{D}$, the corresponding message spaces are publicly known (since $\mathcal{M}, p k_{0}$ and $p k_{1}$ are known). Hence for satisfying Def. 2, it is required that all message spaces should be sufficiently large so that it is negligible for $\mathcal{D}$ to guess correctly the message. One may consider that every message space should have at least $2^{k}$ messages. We will give a more precise specification to the message space. One should also note that the size of the message space is a necessary requirement to the anonymity of a signature scheme, but it is not sufficient.

On the signature spaces, Def. 2 also indicates that $\mathcal{D}$ should not be able to distinguish computationally a signature from one space to another. As a counterexample, if the signature space correlates to the length of the corresponding public key (mentioned earlier in the introduction section), $\mathcal{D}$ may be able to compromise the anonymity of a signature from this information.

\section{Signature Signatures That Are Not Signer Anonymous}

\subsection{The Basic RSA Signature Scheme}

In the following, we show that unless intentionally specified, the basic RSA signature scheme [15] (the primitive one without using hash function), in its general use, is not signer anonymous according to Def. 2.

Consider two signers Signer $_{0}$ and Signer $_{1}$ with RSA moduli $N_{0}$ and $N_{1}$, respectively. Without loss of generality, let $N_{0}>N_{1}$. If the two moduli are of different length, it is obvious that signatures generated by the two signers can easily be identified by checking the length of a given signature. Even if $N_{0}$ and $N_{1}$ are of equal length, we can still distinguish signatures for most of the cases. In the following, we elaborate this in detail.

Let us evaluate the probability that a signature of Signer $_{0}$ falls into the range of $\mathbb{Z}_{N_{0}}-\mathbb{Z}_{N_{1}}$. Let $\Delta=N_{0}-N_{1}$. The probability that a signature of Signer $_{0}$ falls into $\left\{N_{1}, \cdots, N_{0}-1\right\}$ will be $\Delta / N_{0}$. This value is upper bounded by $\Delta / 2^{k-1}$ if $\left|N_{0}\right|=k$. Hence if $|\Delta|$ is in the order of $\log (k)$, then the probability will be negligible for sufficiently large $k$. This is the case when we say that $N_{0}$ and $N_{1}$ are "very close" to each other. In this case, the basic RSA signature scheme may be anonymous. However, this is true only if all message spaces in the system are dense in the corresponding ranges, for example, every element in $\mathbb{Z}_{N_{i}}, i=0,1$, is valid/meaningful. On the other hand, if the message space of Signer $_{0}$ or Signer $_{1}$ is sparse in $\mathbb{Z}_{N_{i}}, i=0 / 1$, that is, there are only a few elements in $\mathbb{Z}_{N_{i}}$ that are valid (or meaningful) messages. Then the scheme cannot be anonymous. 
For example, suppose a signature $\sigma=m_{0}^{d_{0}} \bmod N_{0}$ is given where $d_{0}$ is the private exponent of Signer $_{0}$, the distinguisher $\mathcal{D}$ can determine if Signer $_{1}$ is the actual signer by computing $m^{\prime}=\sigma^{e_{1}} \bmod N_{1}$, where $e_{1}$ is the public exponent of Signer $_{1}$ and then determining if $m^{\prime}$ is in the message space of Signer $_{1}$. Since the message space of Signer $_{1}$ in $\mathbb{Z}_{N_{1}}$ is sparse, it will have a non-negligible chance that $m^{\prime}$ is not in the message space, which allows $\mathcal{D}$ to find out the actual signer with non-negligible advantage.

All of the above are concerning about special cases. In the general case where $N_{0}$ and $N_{1}$ are generated by following a conventional procedure, that is, each of $N_{0}$ and $N_{1}$ is a product of two randomly chosen equal-length primes and $\left|N_{0}\right|=\left|N_{1}\right|=k$, the following theorem implies that with at least a constant probability that a RSA signature can be distinguished successfully (i.e. not signer anonymous under Def. 2).

Theorem 1. If $N_{0}$ and $N_{1}$ are generated by following the conventional procedure, then the probability that $\left|N_{0}-N_{1}\right| \geq 2^{k-2}$ is at least $\frac{1}{400}$.

Due to page limitation, readers please refer to our full paper [19] for the proof.

PSS. Based on the results above, we can see that PSS [5] is not signer anonymous either. Due to page limitation, readers please refer to our full paper [19] for details.

\subsection{Schnorr Signature Scheme [16]}

On input a security parameter $1^{k}$, the key generation algorithm $\mathcal{K}$ returns a public key $p k$ which consists of a set of group parameters $\mathcal{I}=(p, q, g, G, h)$ and an element $y \in G$, and a secret key $s k$ which is a random element $x \in_{R} \mathbb{Z}_{q}$, such that $y=g^{x} \bmod p$. In $\mathcal{I}, p, q$ are two large primes chosen randomly such that $q \mid p-1, G$ is a subgroup of $\mathbb{Z}_{p}^{*}$ with order $q, g$ is a generator of $G$ so that computing discrete logarithms to the base $g$ is difficult, and $h:\{0,1\}^{*} \rightarrow\left\{0,1, \cdots, 2^{k}-1\right\}$ is a hash function where $2^{k}<q$.

In the original Schnorr signature scheme, the message space can be arbitrarily specified as any subset of $\{0,1\}^{*}$. For allowing us to specify the minimum size of the message space that an anonymous Schnorr signature scheme should be in the later part of this paper, we quantify the message space. We define the message space generator $\mathcal{M}$ such that on input $p k$, which is generated by $\mathcal{K}\left(1^{k}\right), \mathcal{M}(p k)$ outputs the description of a message space $M^{\text {Schnorr }}$ such that $\left|M^{\text {Schnorr }}\right| \geq 2^{k}$. Below are the signature generation and verification algorithms.

Signing algorithm. On input a message $m \in M^{\text {Schnorr }}$ and a secret key $x, \mathcal{S}(m, x)$ is computed as follows:

1. Choose a random $w \in_{R} \mathbb{Z}_{q}$ and compute $t=g^{w} \bmod p$.

2. Compute $r=h(t, m)$.

3. Compute $s=w-x r \bmod q$.

The signature for $m$ is the pair $(r, s)$. 
Verification algorithm. To verify a signature $(r, s)$ for message $m$ under public key $(\mathcal{I}, y)$, compute $t=g^{s} y^{r} \bmod p$ and output 1 if $r=h(t, m)$, otherwise output 0 .

Since signers generate their public key pairs independently, it is pretty likely that different signers have their keys under different sets of group parameters. We can see that the scheme is not signer anonymous as identity information will be leaked from the value of $s$ by applying similar arguments to that in Sec. 4.1. Interestingly, in a special case where all signers are sharing a common set of group parameters, the scheme can actually be shown to provide signer anonymity under the random oracle model 4 without any modification. The proof technique is similar to that for Lemma 2

\section{An Adaptive Security Model for Signer Anonymity}

Def. 2 is static as the distinguisher cannot adaptively acquire additional information about the challenging signature from the environment. In the following, we define a stronger model which allows the distinguisher to adaptively obtain signatures generated by the entity who generates the challenging signature.

Definition 3 (SA-CMA). Let $k$ be a security parameter. A digital signature scheme $\mathcal{S D}$ is signer anonymous against chosen message attack (SA-CMA) if for all sufficiently large $k$, no PPT adversary (or distinguisher) $\mathcal{D}$ can win the following game with a probability non-negligibly larger than $\frac{1}{2}$. The game is simulated by a challenger.

1. (Key Generation Phase.) The challenger runs $\mathcal{K}\left(1^{k}\right)$ multiple times for generating polynomially many public and secret key pairs. All the public keys are accessible by $\mathcal{D}$.

2. (Training Phase.) $\mathcal{D}$ adaptively queries the challenger with a public key $p k_{i}$ and a message $m \in \mathcal{M}\left(p k_{i}\right)$. The challenger produces $\sigma \leftarrow \mathcal{S}\left(m, s k_{i}\right)$ and replies $\mathcal{D}$ with $\sigma$ if $p k_{i}$ is generated in the Key Generation Phase; otherwise, $a$ ' $\perp$ ' is returned indicating that signature generation has failed.

3. (Key Selection Phase I.) D picks two public keys from the public keys generated in the Key Generation Phase. We denote these two key pairs by $\left(p k_{0}, s k_{0}\right)$ and $\left(p k_{1}, s k_{1}\right)$.

4. (Key Selection Phase II.) The challenger gives all the secret keys to $\mathcal{D}$ except $s k_{0}$ and $s k_{1}$.

5. (Challenge Phase.) The challenger tosses a random coin $\varpi \stackrel{R}{\leftarrow}\{0,1\}$, then uniformly picks a message $m \in \mathcal{M}\left(p k_{\varpi}\right)$, and returns a challenge signature $\sigma \leftarrow \mathcal{S}\left(m, s k_{\varpi}\right)$ to $\mathcal{D}$.

6. (Cracking Phase.) $\mathcal{D}$ can still adaptively make signing queries as in the Training Phase but the associated public key with each query can only be $p k_{0}$ or $p k_{1}$.

7. (Output Phase.) At the end of the game, $\mathcal{D}$ outputs a bit $\varpi^{\prime}$ and wins if $\varpi^{\prime}=\varpi$. 
$\mathcal{D}$ 's advantage is defined as $\mathbf{A d v}^{\text {sa-cma }}=\operatorname{Pr}\left[\varpi^{\prime}=\varpi\right]-\frac{1}{2}$ and $\operatorname{Pr}\left[\varpi^{\prime}=\varpi\right]$ is the probability that $\mathcal{D}$ wins the game. The probability is taken over the coin tosses of both $\mathcal{D}$ and the challenger, including the coin toss for $\varpi$.

If a scheme satisfies this definition, we say that the scheme is SA-CMA secure. Note that in the Cracking Phase we only allow the distinguisher to query with public key $p k_{0}$ or $p k_{1}$, since the secret keys corresponding to all other public keys have already been given to the distinguisher.

As the distinguisher $\mathcal{D}$ of the adaptive model has an additional signing oracle to access, the model is obviously stronger than the static one given in Def. 2, Another seemingly "stronger" definition is to let $\mathcal{D}$ perform the Challenge Phase and the Cracking Phase in the following way:

\section{Definition 4.}

5. The challenger tosses a random coin $\varpi \stackrel{R}{\leftarrow}\{0,1\}$.

6. $\mathcal{D}$ can adaptively perform the following queries:

(a) $\mathcal{D}$ performs signing queries as in the Training Phase except that now the allowable public keys are $p k_{0}$ and $p k_{1}$ only.

(b) $\mathcal{D}$ queries a special oracle called challenging oracle. The challenging oracle uniformly picks a message $m \in \mathcal{M}\left(p k_{\varpi}\right)$, and returns $\sigma \leftarrow \mathcal{S}\left(m, s k_{\varpi}\right)$ to $\mathcal{D}$.

...

But the following result shows that Def. 3 and Def. 4 are equivalent.

Theorem 2. If there exists no PPT algorithm that has a non-negligible advantage in winning the game in Def. 國, then there exists no PPT algorithm that has a non-negligible advantage in winning the game in Def. 因.

Due to page limitation, readers please refer to our full paper [19] for the proof.

\section{Modified Signature Schemes for Signer Anonymity}

In this section, we propose some modifications on the schemes described in Sec.4 and show that they are signer anonymous under the adaptive model (i.e. SACMA in Def. 3). We start with Schnorr signature scheme and provide the full proof for its signer anonymity. Then we modify the basic RSA signature scheme and subsequently the PSS. Due to page limitation, readers please refer to our full paper 19 for the discussions of the last two schemes.

Extended Schnorr Signature Scheme for Signer Anonymity. The key generation algorithm $\mathcal{K}$ and the message space generator $\mathcal{M}$ are almost the same as the original Schnorr signature scheme described in Sec. 4.2, except that the public key now also contains an additional parameter denoted by $b \in \mathbb{N}$. Let $q_{\min }$ and $q_{\max }$ denote the lower bound and upper bound of the group orders of all signers, respectively. Let $2^{b}$ be an integer which is $\ell$ bits longer than $q_{\max }$ and $\ell=k+1$. One may imagine $k=160$ and hence $\ell=161$. Let $h:\{0,1\}^{*} \rightarrow$ $\left\{0,1, \cdots, 2^{k}-1\right\}$ be a hash function where $2^{k}<q_{\text {min }}$. 
For a signer with public key $p k=(\mathcal{I}, b, y)$ and secret key $x$ generated by $\mathcal{K}\left(1^{k}\right)$ where $\mathcal{I}=(p, q, g, G, h)$ and $y=g^{x} \bmod p$, the signature generation and verification algorithms are as follows. Let $n$ be the largest integer such that $n q<2^{b}$.

Signing algorithm. On input a message $m \in \mathcal{M}(p k)$ and secret key $x, \mathcal{S}(m, x)$ is computed as follows:

1. Choose a random $w \in \mathbb{Z}_{q}$ and compute $t=g^{w} \bmod p$.

2. Compute $r=h(t, m)$ and then $s=w-x r \bmod q$.

3. Choose a number $\lambda \stackrel{R}{\leftarrow}\{0,1, \cdots, n-1\}$ and compute $s^{\prime}=s+\lambda q$ The signature for $m$ is the pair $\left(r, s^{\prime}\right)$.

Verification algorithm. To verify signature $\left(r, s^{\prime}\right)$ for message $m$ and public key $(\mathcal{I}, y)$, compute $s=s^{\prime} \bmod q$ and $t=g^{s} y^{r} \bmod p$, and output 1 if $r=h(t, m)$, otherwise, output 0 .

Consider two arbitrary signers Signer $_{i}$ and Signer $_{j}$ whose sets of group parameters are denoted by $\mathcal{I}_{i}=\left(p_{i}, q_{i}, g_{i}, G_{i}, h\right)$ and $\mathcal{I}_{j}=\left(p_{j}, q_{j}, g_{j}, G_{j}, h\right)$, respectively. Let $n_{i}$ and $n_{j}$ be the largest integers such that $n_{i} q_{i}<2^{b}$ and $n_{j} q_{j}<2^{b}$, respectively. Without loss of generality, we assume $n_{i} q_{i}<n_{j} q_{j}$.

Lemma 1. For the extended Schnorr signature scheme above, if signer Signer $_{i}$ generates a signature $\left(r_{i}, s_{i}^{\prime}\right)$ and signer Signer $_{j}$ generates a signature $\left(r_{j}, s_{j}^{\prime}\right)$, then the probability that $s_{j}^{\prime}$ is in $\Delta=\left\{n_{i} q_{i}, \cdots, n_{j} q_{j}-1\right\}$ is at most $2^{-k}$.

Proof. First, note that $s_{i}^{\prime}$ and $s_{j}^{\prime}$ are uniformly distributed on $\left\{0,1, \cdots, n_{i} q_{i}-1\right\}$ and $\left\{0,1, \cdots, n_{j} q_{j}-1\right\}$, respectively. Second, since $n_{j} q_{j}<2^{b}$ and $n_{i} q_{i} \geq 2^{b}-q_{i}$, $n_{j} q_{j}-n_{i} q_{i}<2^{b}-\left(2^{b}-q_{i}\right)=q_{i} \leq q_{\max }$. Hence,

$$
\operatorname{Pr}\left[s_{j}^{\prime} \in \Delta\right]<q_{\max } /\left(2^{b}-q_{\max }\right)<1 / 2^{l-1}=1 / 2^{k} .
$$

In the following, we assume that $h$ behaves like a random oracle [4. If an algorithm $\mathcal{A}$ runs in time at most $t$ and completes successfully with probability at least $\epsilon>0$, then $\mathcal{A}$ is said to be a $(t, \epsilon)$-algorithm. The probability is taken over the input domain and the coin tosses of $\mathcal{A}$.

Lemma 2. In the extended Schnorr signature scheme above, suppose for any pair of signers Signer Sind $_{i}$ Signer ${ }_{j}, q_{i}=q_{j}$. Then if there exists a $\left(t, \epsilon+\frac{1}{2}\right)$ algorithm (distinguisher) $\mathcal{D}$ which wins the game of Def. 3 after performing at most $q_{H}$ hash queries and $q_{S}$ signing queries, there exists a $\left(t^{\prime}, \epsilon^{\prime}\right)$-algorithm $\mathcal{F}$ which existentially forges under the chosen message attack [9] a signature after performing at most $q_{H}+q_{S}$ hash queries and $q_{S}$ signing queries, where $t^{\prime} \leq t+q_{K} c$ and $\epsilon^{\prime} \geq\left(1-\frac{q_{H}+q_{S}}{2^{k}}\right)\left(1-\frac{q_{S}}{2^{k}}\right) \frac{\epsilon}{q_{K}}$ for $q_{K}$ being some polynomial in $k$ and $c$ being the time required for generating one key pair in the extended Schnorr signature scheme.

Proof. We construct an algorithm $\mathcal{F}$ which runs $\mathcal{D}$ under a simulated environment of Def. 3 and forges a Schnorr signature. 
At the beginning of the simulation, $\mathcal{F}$ is given a security parameter $k$, a set of group parameters $\mathcal{I}=(p, q, g, G, h)$, a challenge element y $\in G$, an auxiliary parameter $b \in \mathbb{N}$ and a message space $M^{\text {Schnorr }}$ such that $\left|M^{\text {Schnorr }}\right| \geq 2^{k}$. $\mathcal{F}$ is to forge a signature $\sigma^{*}=\left(r^{*}, s^{*}\right)$ with message $m^{*} \in M^{\text {Schnorr }}$ such that $r^{*}=h\left(g^{s^{*}} \mathrm{y}^{r^{*}} \bmod p, m^{*}\right)$ where $h$ is provided as a random oracle by the unforgeability game simulator of $\mathcal{F}$. Note that $\mathcal{F}$ has access to the random oracle of $h$ and a signing oracle corresponding to the challenge public key y. The signing oracle, on input a message $m \in M^{\text {Schnorr }}$, returns a signature $\sigma=(r, s)$ such that $r=h\left(g^{s} \mathrm{y}^{r} \bmod p, m\right)$. We denote the random oracle for $h$ by $\mathcal{H O}$ and the signing oracle by $\mathcal{S O}$.

In the Key Generation Phase of the game defined in Def. $3, \mathcal{F}$ randomly generates $q_{K}-1$ public key pairs where $q_{K}$ is some polynomial in $k$. For each of the public key pairs, say the $i$-th, the set of group parameters $\mathcal{I}_{i}=\left(p_{i}, q_{i}, g_{i}, G_{i}, h\right)$ is generated such that $q_{i}=q, q_{i} \mid p_{i}-1$, and $g_{i}$ is the generator of $G_{i}$ whose order is $q_{i}$. Also an element $y_{i}$ is generated as $g_{i}^{x_{i}} \bmod p_{i}$ where $x_{i}$ is randomly chosen from $\mathbb{Z}_{q_{i}}$. The public key of $i$-th public key pair is set to $p k_{i}=\left(\mathcal{I}_{i}, b, y_{i}\right)$ and the corresponding secret key is $x_{i}$. Let $\mathcal{L}=\left\{p k_{i}\right\}_{1 \leq i \leq q_{K}}$ be the set of public keys generated in this phase except $p k_{j}$, which instead is assigned to $(\mathcal{I}, b, \mathrm{y})$. The value of $j$ is chosen randomly from 1 to $q_{K}$.

In the Training Phase and the Cracking Phase, $\mathcal{F}$ answers all oracle queries made by $\mathcal{D}$. For a hash query, the query is relayed by $\mathcal{F}$ to $\mathcal{H O}$ for an answer. The answer is then relayed back to $\mathcal{D} . \mathcal{F}$ also maintains a list $\Psi$ of queried values and their returns. For a signature query with message $m$ in the corresponding message space, there are two cases. Case 1: if the public key is not y, $\mathcal{F}$ follows the signing algorithm of the scheme to generate a signature. This can be done as $\mathcal{F}$ knows the corresponding signing key (or secret key). Case 2: if the public key is y, $\mathcal{F}$ relays the query to $\mathcal{S O}$ and relays the signature back to $\mathcal{D}$. Note that the list $\Psi$ should also be updated for hash values. In addition to these steps, in the Cracking Phase, we will see shortly that $\mathcal{F}$ needs to carry out a few more checkings when relaying queries and answers between $\mathcal{D}$ and the oracles $\mathcal{H O}$, $\mathcal{S O}$ to and fro.

In the Key Selection Phase I, if $\mathcal{D}$ picks two public keys such that none of the keys is y, $\mathcal{F}$ fails and halts. Let the two public keys be $\left(\hat{\mathcal{I}}_{0}, b, \hat{y}_{0}\right),\left(\hat{\mathcal{I}}_{1}, b, \hat{y}_{1}\right)$. Suppose $\mathcal{F}$ does not fail and proceeds successfully to the Challenge Phase, $\mathcal{F}$ sets the challenge signature $\sigma^{*}=\left(r^{*}, s^{*}\right)$ by randomly picks $r^{*} \stackrel{R}{\leftarrow}\{0,1\}^{k}$ and $s^{*} \stackrel{R}{\leftarrow}\{0,1, \cdots, n q-1\}$ where $n$ is the largest integer so that $n q<2^{b}$. If $r^{*}$ is already in the list $\Psi$ as a queried hash oracle answer, $\mathcal{F}$ fails and halts (we will see below that this event is called $\left.\mathbf{E}_{2}\right)$. Otherwise, an entry $\left(\top, r^{*}\right)$ is added into the list $\Psi$, where $T$ represents some hash input whose value is not known yet but its hash value has been given as $r^{*}$.

The simulation proceeds until $\mathcal{D}$ reaches the Output Phase. When $\mathcal{D}$ outputs and halts, $\mathcal{F}$ also halts and outputs nothing. That means $\mathcal{F}$ has failed to forge a signature. However during the Cracking Phase, whenever $\mathcal{D}$ makes a hash query, $\mathcal{F}$ checks if the answer of $\mathcal{H O}$ is $r^{*}$. If this is the case and at the same time the hash evaluation is of the form $h\left(g^{s^{*}} \mathrm{y}^{r^{*}} \bmod p, m^{*}\right)$ where $m^{*} \in M^{\text {Schnorr }}$ 
and $m^{*}$ is not involved in a signing query in the Training phase, $\mathcal{F}$ outputs the forged signature $\sigma^{*}=\left(r^{*}, s^{*}\right)$ and message $m^{*}$, and halts. In addition, during the Cracking Phase, whenever $\mathcal{D}$ makes a signing query with some message $m^{*} \in M^{\text {Schnorr }}$ under y, $\mathcal{F}$ first queries $\mathcal{H O}$ for the value of $h\left(g^{s^{*}} \mathrm{y}^{r^{*}} \bmod p, m^{*}\right)$. If the hash value is equal to $r^{*}$ and $m^{*}$ is not involved in a signing query in the Training Phase, $\mathcal{F}$ outputs the forged signature $\sigma^{*}=\left(r^{*}, s^{*}\right)$ and message $m^{*}$, and halts; if the hash value is not $r^{*}, \mathcal{F}$ then relays the query to $\mathcal{S O}$ and continues the simulation as described above. Note that if $m^{*}$ turns out to have been queried in some signing query during the Training Phase, $\mathcal{F}$ fails and halts (we will see below that this event is called $\mathbf{E}_{3}$ ).

Analysis. First of all, it is easy to see that the running time of $\mathcal{F}$ is in polynomial of that of $\mathcal{D}$ and $\mathcal{F}$ perfectly simulates the game of Def. 3 except during the Challenge Phase. In this phase, the challenger in a real game (that is, $\mathcal{F}$ in the simulated game described above) should have randomly picked a key among two given public keys, then picked a message randomly from the message space corresponding to the chosen public key and generated a challenge signature accordingly.

First, we investigate the distribution of the messages which produce a signature $\left(r^{*}, s^{*}\right)$ with respect to each of $\left(\hat{\mathcal{I}}_{0}, b, \hat{y}_{0}\right)$ and $\left(\hat{\mathcal{I}}_{1}, b, \hat{y}_{1}\right)$. For each of $\left(\hat{\mathcal{I}}_{\varpi^{*}}, b, \hat{y}_{\varpi^{*}}\right), \varpi^{*}=0,1$, define two sets

$$
M_{\varpi^{*}}=\left\{m: r^{*} \leftarrow h\left(g_{\varpi *}^{s^{*}} \hat{y}_{\varpi *}^{r^{*}} \bmod p_{\varpi *}, m\right), m \in M_{\varpi^{*}}^{S c h n o r r}\right\} .
$$

Under the assumption that $h$ is a random function [4], $M_{\varpi^{*}}$ is uniformly distributed, and the expected number of messages in $M_{\varpi^{*}}$ is equal to $\left|M_{\varpi^{*}}^{S c h n o r r}\right| / 2^{k}$. From the fact that $\log _{2}\left(\left|M_{\varpi^{*}}^{S c h n o r r}\right|\right) \geq k$, we have at least half chance (derived from $\left.1-\left(1-2^{-k}\right)^{\left|M^{\text {Schnorr }} \varpi^{*}\right|} \geq 1 / 2\right)$ that the challenge signature $\sigma^{*}=\left(r^{*}, s^{*}\right)$, generated by $\mathcal{F}$ in the Challenge Phase of the simulated game above, is a valid signature of some message in $M_{\varpi^{*}}^{S c h n o r r}$.

Let $\mathbf{E}_{1}$ be the event that the hash evaluation

$$
r^{*} \leftarrow h\left(g_{\varpi *}^{s^{*}} \hat{y}_{\varpi *}^{r^{*}} \bmod p_{\varpi *}, m^{*}\right)
$$

is carried out during the cracking phase where $\varpi^{*}=0 / 1$. If event $\mathbf{E}_{1}$ does not occur, it is indistinguishable from $\mathcal{D}$ 's point of view between the Challenge Phase of a real game and that of the simulated game by $\mathcal{F}$. By the random oracle assumption, it is unknown on which message $m^{*}$ will make Eq. (1) hold. Hence $\mathcal{D}$ has no advantage in winning the game.

Since the position of $(\mathcal{I}, b, y)$ in $\mathcal{L}$ is randomly chosen, the probability of selecting $(\mathcal{I}, b, y)$ in Key Selection Phase I is $2 / q_{K}$. Due to the same reason, in event $\mathbf{E}_{1}$, the chance that $\hat{y}_{\varpi *}=\mathrm{y}$ is $1 / 2$. Note that $\operatorname{Pr}[\mathcal{D}$ wins $] \geq \epsilon+1 / 2$. Let $\operatorname{Pr}\left[\mathcal{D}\right.$ wins $\left.\mid \mathbf{E}_{1}\right]=\lambda+1 / 2$. We have

$$
\begin{aligned}
\epsilon+\frac{1}{2} & \leq \operatorname{Pr}[\mathcal{D} \text { wins }] \\
& =\left(\lambda+\frac{1}{2}\right) \operatorname{Pr}\left[\mathbf{E}_{1}\right]+\operatorname{Pr}\left[\mathcal{D} \text { wins } \mid \overline{\mathbf{E}_{1}}\right] \operatorname{Pr}\left[\overline{\mathbf{E}_{1}}\right]
\end{aligned}
$$




$$
=\left(\lambda+\frac{1}{2}\right) \operatorname{Pr}\left[\mathbf{E}_{1}\right]+\frac{1}{2} \operatorname{Pr}\left[\overline{\mathbf{E}_{1}}\right] .
$$

Hence $\lambda \operatorname{Pr}\left[\mathbf{E}_{1}\right] \geq \epsilon$. Since $\epsilon>0$, we have $0<\lambda \leq 1 / 2$. Therefore $\operatorname{Pr}\left[\mathbf{E}_{1}\right] \geq 2 \epsilon$.

To find out the lower bound of the winning probability of $\mathcal{F}$, we only have two events left to evaluate, that is, the chance that $\mathcal{F}$ fails due to the following two events.

Event $\mathbf{E}_{2}$ : During the Challenge Phase, $r^{*}$ is found to be in the list of $\Psi$. Event $\mathbf{E}_{3}$ : During the Cracking Phase, if evaluation $r^{*} \leftarrow h\left(g^{s^{*}} \mathrm{y}^{r^{*}} \bmod \right.$ $\left.p, m^{*}\right)$ occurs while $m^{*}$ has been involved in a signing query during the Training Phase.

Since $r^{*}$ is randomly chosen from $\{0,1\}^{k}$ and $h$ is a random function, we have $\operatorname{Pr}\left[\mathbf{E}_{2}\right] \leq \frac{q_{H}+q_{S}}{2^{k}}$. Similarly, we have $\operatorname{Pr}\left[\mathbf{E}_{3}\right] \leq \frac{q_{S}}{2^{k}}$.

Combining all the events above, they include the case that $\mathrm{y}$ is one of $\hat{y}_{0}$ and $\hat{y}_{1}$, the case that $\left(r^{*}, s^{*}\right)$ is a valid signature of $\mathbf{y}, \mathbf{E}_{1}$ occurs, the case that $\mathrm{y}$ is involved in the event $\mathbf{E}_{1}$, the case that $r^{*}$ is not in the list $\Psi$ during the Challenge Phase (i.e. $\overline{\mathbf{E}_{2}}$ ), and the case that the forged message $m^{*}$ has not been involved in any signing query during the Training Phase (i.e. $\overline{\mathbf{E}_{3}}$ ), we have

$$
\operatorname{Pr}[\mathcal{F} \text { wins }] \geq\left(1-\frac{q_{H}+q_{S}}{2^{k}}\right)\left(1-\frac{q_{S}}{2^{k}}\right) \frac{\epsilon}{q_{K}} .
$$

On the running time of $\mathcal{F}$, we can see that besides running $\mathcal{D}, \mathcal{F}$ needs to generates $q_{K}-1$ key pairs during the Key Generation Phase and at most $q_{S}$ additional hash queries during the Cracking Phase. Let $c$ be the time required for generating one key pair. The running time of $\mathcal{F}$ is at most $t+q_{K} c$. Also $\mathcal{F}$ performs at most $q_{H}+q_{S}$ hash queries and $q_{S}$ signing queries.

Theorem 3. The extended Schnorr signature scheme described above is SACMA secure.

Proof. Without loss of generality, suppose in the game of Def. 3, the distinguisher $\mathcal{D}$ picks the public keys corresponding Signer $_{i}$ and Signer $_{j}$ in the Key Selection Phase I, and Signer ${ }_{j}$ is picked by the challenger in the Challenge Phase. We follow the notations used above and in the proof of Lemma 1, we assume that $n_{i} q_{i}<n_{j} q_{j}$. Let $\mathbf{E}$ be the event that $s_{j}^{\prime} \notin \Delta$. In other words, $\mathbf{E}$ is the event that $s_{j}^{\prime} \in\left\{0,1, \cdots, n_{i} q_{i}-1\right\}$, that is, in the same domain as Signer $_{i}$ has been picked by the challenger. According to Lemma 2 we have $\operatorname{Pr}[\mathcal{D}$ wins the game $\mid \mathbf{E}] \leq$ $\frac{1}{2}+\epsilon(k)$ under the assumption that the extended Schnorr signature scheme is existentially unforgeable [9, where $\epsilon$ is a negligible function. Since $\operatorname{Pr}[\mathbf{E}] \leq 1$, we have

$$
\operatorname{Pr}[\mathcal{D} \text { wins the game } \wedge \mathbf{E}] \leq \frac{1}{2}+\epsilon(k)
$$

According to Lemma1, we have $\operatorname{Pr}[\overline{\mathbf{E}}] \leq 2^{-k}$. Since $\operatorname{Pr}[\mathcal{D}$ wins the game $\mid \overline{\mathbf{E}}] \leq 1$, we have

$$
\operatorname{Pr}[\mathcal{D} \text { wins the game } \wedge \overline{\mathbf{E}}] \leq 2^{-k}
$$


Combining Eq. (2) and (3), we have

$$
\operatorname{Pr}[\mathcal{D} \text { wins the game }] \leq \frac{1}{2}+\epsilon(k)+2^{-k}
$$

The extended Schnorr signature scheme still maintains existential unforgeability against adaptive chosen message attack (euf-cma) [9], namely, given a signing oracle, an adversary cannot forge a signature for a message $m$ which has not been queried to the signing oracle before. However, the extended scheme does not satisfy the strong unforgeability [3,1], namely, given a signing oracle, an adversary cannot forge a valid pair of message $m$ and signature $\sigma$ which has not been a query output of the signing oracle for $m$ before.

\section{Applications}

\subsection{Anonymous Key Exchange}

As shown in Fig. 1 1 and discussed in the introduction section, the protocol cannot provide client anonymity if the Different Domain Attack is feasible. In order to make it client anonymous, we modify the last message flow from $A$ to $B$ by using an anonymous signature scheme and change the message to

$$
A \rightarrow B: \operatorname{Sig}_{A}\left(h\left(I D_{B}, \text { count }, \sigma, r_{B}\right)\right)
$$

where $h:\{0,1\}^{*} \rightarrow\{0,1\}^{k}$ is a hash function which behaves like a random oracle.

The example above is an anonymous key transport protocol. Next, we construct an anonymous key exchange protocol which not only ensures the anonymity of the client but also allows the client and the server to establish a session key from both of their session key contributions. The protocol is based on a key exchange protocol called "SIG-DH" [8] which is a signature-based variation of the Diffie-Hellman key exchange protocol with provable security against various active attacks defined in the Canetti-Krawczyk model [8].

Let $k$ be a security parameter. Let $G$ be a group generated by $g$ with large prime order $q$ so that computing discrete logarithms to be base $g$ is difficult. Let $H:\{0,1\}^{*} \rightarrow\{0,1\}^{3 k}$ be a hash function. Each party has a secret signing key for a signature algorithm $\operatorname{Sig}$. By $\operatorname{Sig}_{A}(m)$, we mean the signature on message $m$ generated by party $A$ with identity $I D_{A} \in\{0,1\}^{k}$. Assume the public keys of all parties in the system are publicly known. Let $E$ be a block cipher (e.g. AES [14]) of block size $k$. Suppose a client (the initiator) $A$ and a server (the responder) $B$ already have a session-id $s$ shared. We will explain shortly on how the session-id $s$ is established. The following protocol is carried out between them.

1. A randomly chooses a temporal identity alias $\in_{R}\{0,1\}^{k}, x \in_{R} \mathbb{Z}_{q}$, and sends (alias, $s, \alpha=g^{x}$ ) to $B$.

2. Upon receipt of (alias, $s, \alpha), B$ randomly chooses $y \in \in_{R} \mathbb{Z}_{q}$, then computes $\kappa_{1}\left\|\kappa_{2}\right\| \kappa_{3} \leftarrow H\left(\alpha^{y}\right)$ such that $\left|\kappa_{i}\right|=k$ for $i=1,2,3$, erases $y$, and sends to $A$ the message $\left(B, s, \beta=g^{y}\right)$ together with $S I G_{B}(B, s, \beta$, $\alpha$, alias $)$. 
3. Upon receipt of $\left(B, s, \beta=g^{y}\right)$ and $B$ 's signature, $A$ computes $\kappa_{1}^{\prime}\left\|\kappa_{2}^{\prime}\right\| \kappa_{3}^{\prime} \leftarrow$ $H\left(\beta^{x}\right)$, erases $x$, and verifies the signature. If the signature is valid, $A$ sends to $B$ the message (alias, $s, C_{1}=E_{\kappa_{1}}(A)$ ) together with its signature $\sigma=$ $\operatorname{Sig}_{A}\left(h\left(\right.\right.$ alias $\left.\left., A, s, \alpha, \beta, B, \kappa_{2}^{\prime}\right)\right)$ where $h:\{0,1\}^{*} \rightarrow\{0,1\}^{k}$ is a hash function. $A$ outputs the session key $\kappa_{3}^{\prime}$ under session-id $s$.

4. Upon receipt of (alias, $\left.s, C_{1}\right)$ and a signature $\sigma, B$ computes $A^{\prime}=E_{\kappa_{1}}^{-1}\left(C_{1}\right)$, and verifies the identity $A^{\prime}$ (e.g. for access control) and signature $\sigma$. If all verifications are passed, $B$ outputs the session key $\kappa_{3}$ under session-id $s$.

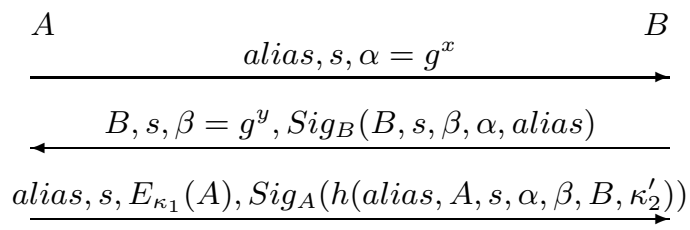

Fig. 2. Anonymous SIG-DH Protocol

(Analysis.) The protocol described above (Fig. 2) supports anonymity of the client $A$ if $\mathrm{Sig}$ is an anonymous signature scheme. In the protocol, all hash functions are assumed to behave like random oracles. The session-id $s$ should also be randomly selected each time for ensuring $A$ 's anonymity. As suggested by the authors of [8], in practice, the session-id $s$ can be a pair $\left(s_{1}, s_{2}\right)$ where $s_{1}$ is a value randomly chosen by $A$ such that it is different from the values in other of $A$ 's sessions and $s_{2}$ is randomly chosen by $B$ in a similar way. These values can be exchanged by the parties as a prologue [10. Alternatively, $s_{1}$ can be included by $A$ in the first message of the protocol, and $s_{2}$ be included by $B$ in the second message.

The protocol assumes that the signature verification keys of all parties are publicly known. In practice, we can add the client's certificate into the encryption in the third message provided that the certificates of all clients are of the same length. Also, we assume that the server does not know the client at the beginning of the communication. In case it is already known, the encryption operation in the third message can be removed from the protocol.

Comparing with the original "SIG-DH" protocol [8], the anonymous version proposed above has an additional message component $\kappa_{2}^{\prime}$ in the signature of $A$. $\kappa_{2}^{\prime}$ is used for satisfying the anonymity requirement of an anonymous signature scheme, that is, preventing an adversary from compromising $A$ 's anonymity by searching through the list of all possible 'messages' of the signature.

\subsection{Anonymous Paper Review}

In a conventional anonymous paper review system for a conference, authors separate their authorship information from the paper bodies before submitting them to the conference organizer. The paper bodies are required to be fully anonymous, that is, no author name, affiliation, acknowledgement, or obvious reference should appear in them. The organizer then keeps the authorship information of 
the papers secret from the reviewers and only sends those anonymized paper bodies to reviewers to review. One problem of the current system is that the anonymity of the papers will be compromised once the authorship information of the papers is leaked to the reviewers from the organizer. The organizer or some insider in the organizing institute, for example a graduate student who is responsible for maintaining the paper submission server, may leak the authorship information of the papers to the reviewers. In the following, we describe a method which uses anonymous signature to solve this collusion problem.

Consider the paper submission server is now a bulletin board which posts and timestamps any message received. Once posted, the message cannot be altered. Let $\operatorname{Paper}_{A}$ be a paper which is fully anonymous. Let $A$ be the identity of the paper's author and assume that each author already has his public key (for signature verification) published. To submit the paper $\operatorname{Paper}_{A}$, the author randomly picks a long binary string $r \in\{0,1\}^{k}$ where $k$ is the security parameter, and generates a signature $\sigma_{A}=\operatorname{AnonSig}_{A}\left(h\left(\operatorname{Paper}_{A}, r\right)\right)$ using his anonymous signature generation algorithm denoted by AnonSig ${ }_{A}$ on the message $h\left(\operatorname{Paper}_{A}, r\right)$ where $h:\{0,1\}^{*} \rightarrow\{0,1\}^{k}$ is a hash function which behaves like a random oracle. The author posts Paper $_{A}$ and $\sigma_{A}$ onto the bulletin board for review. When all the reviews are completed and the acceptance decision on each paper has been made, the decision will be posted on the bulletin board. If $\operatorname{Paper}_{A}$ is accepted, the author $A$ will reveal the value of $r$ for claiming his authorship on $\operatorname{Paper}_{A}$. From this point on, everyone is able to verify his authorship using $\sigma_{A}$, $\left(\operatorname{Paper}_{A}, r\right)$ and $A$ 's public key.

Discussions: In the review stage, no author has given out any authorship information and the secrecy of $r$ prevents anyone from identifying the signer of $\sigma_{A}$. This new system can also let the public access the bulletin board instead of restricting its access to reviewers only. In this way, everyone can access those papers once they are posted. Since every paper is timestamped when it is first submitted and posted to the bulletin board, this helps paper authors to claim that they are the first ones who obtained those new results described in their papers without compromising the process of anonymous review. In addition, it will also help discover parallel submissions.

\section{References}

1. J.H. An, Y. Dodis, and T. Rabin. On the security of joint signature and encryption. In Proc. EUROCRYPT 2002, pages 83-107. Springer-Verlag, 2002. LNCS 2332.

2. M. Bellare, A. Boldyreva, A. Desai, and D. Pointcheval. Key-privacy in public-key encryption. In Proc. ASIACRYPT 2001, pages 566-582. Springer-Verlag, 2001. LNCS 2248.

3. M. Bellare and C. Namprempre. Authenticated encryption: Relations among notions and analysis of the generic composition paradigm. In Proc. ASIACRYPT 2000, pages 531-545. Springer-Verlag, 2000. LNCS 1976.

4. M. Bellare and P. Rogaway. Random oracles are practical: A paradigm for designing efficient protocols. In First ACM Conference on Computer and Communications Security, pages 62-73, Fairfax, 1993. ACM. 
5. M. Bellare and P. Rogaway. The exact security of digital signatures - how to sign with RSA and Rabin. In Advances in Cryptology - Eurocrypt'96, pages 399-416. Springer-Verlag, 1996. LNCS 1070.

6. C. Boyd and D. Park. Public key protocols for wireless communications. The 1st International Conference on Information Secuirty and Cryptology (ICISC'98), pages 47-57, 1998.

7. X. Boyen. Multipurpose identity-based signcryption: A swiss army knife for identity-based cryptography. In Proc. CRYPTO 2003, pages 383-399. SpringerVerlag, 2003. LNCS 2729.

8. R. Canetti and H. Krawczyk. Analysis of key-exchange protocols and their use for building secure channels. In Proc. EUROCRYPT 2001, pages 453-474. SpringerVerlag, 2001. LNCS 2045. http://eprint.iacr.org/2001/040/.

9. S. Goldwasser, S. Micali, and R. Rivest. A digital signature scheme secure against adaptive chosen-message attack. SIAM J. Computing, 17(2):281-308, April 1988.

10. D. Harkins, C. Kaufman, and R. Perlman. The internet key exchange (IKE) protocol < draft-ietf-ipsec-ikev2-00.txt $>$. INTERNET-DRAFT, November 2001.

11. E. Van Herreweghen. Secure anonymous signature-based transactions. In ESORICS '00: Proc. of the 6th European Symposium on Research in Computer Security, pages 55-71. Springer-Verlag, 2000. LNCS 1895.

12. M. Jakobsson, K. Sako, and R. Impagliazzo. Designated verifier proofs and their applications. In Proc. EUROCRYPT 96, pages 143-154, 1996. LNCS 1070.

13. F. Laguillaumie and D. Vergnaud. Designated verifier signatures: Anonymity and efficient construction from any bilinear map. In Proc. of the 4th Intl. Conference on Security in Communication Networks (SCN 2004), pages 105-119, 2004. LNCS 3352.

14. NIST FIPS PUB 197. Announcing the ADVANCED ENCRYPTION STANDARD (AES), November 2001.

15. R. Rivest, A. Shamir, and L. Adleman. A method for obtaining digital signatures and public-key cryptosystems. Communications of the ACM, 21(2):120-126, February 1978.

16. C. Schnorr. Efficient identification and signatures for smart cards. In Proc. CRYPTO 89, pages 239-252. Springer, 1990. LNCS 435.

17. G. Yang, D. Wong, and X. Deng. Analysis and improvement of a signcryption scheme with key privacy. In Proc. of the 8th Information Security Conference (ISC '05), pages 218-232. Springer-Verlag, 2005. LNCS 3650.

18. G. Yang, D. Wong, and X. Deng. Efficient anonymous roaming and its security analysis. In Proc. of the 3rd International Conference on Applied Cryptography and Network Security (ACNS 2005), pages 334-349. Springer-Verlag, 2005. LNCS 3531.

19. G. Yang, D. S. Wong, X. Deng, and H. Wang. Anonymous signature schemes. Cryptology ePrint Archive, Report 2005/407, 2005. http://eprint.iacr.org/ 